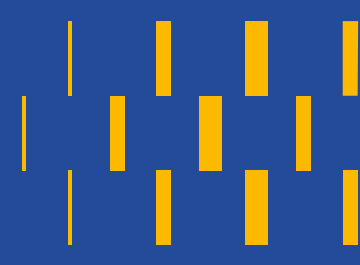

\title{
De extranjeros a ciudadanos urbanos: autoconstrucción y migración en el Gran Santiago
}

\section{From foreigners to urban citizens: autoconstruction and migration in the Santiago Metropolitan Area}

\author{
Miguel Pérez1 (D) https://orcid.org/0000-0002-7523-1116 \\ Cristóbal Palma² (D) https://orcid.org/0000-0002-4590-3145 \\ ${ }^{1}$ Universidad Alberto Hurtado, Santiago, CHILE. Email: miguelperez@uahurtado.cl \\ ${ }^{2}$ Universidad Alberto Hurtado, Santiago, CHILE. Email: cristobal.spr@gmail.com
}

\section{Resumen}

En diversas metrópolis del llamado Sur global, el reclamo por el derecho a habitar en la ciudad ha desembocado en extensos procesos de "autoconstrucción", concepto que alude a un modo de edificar las periferias urbanas en el que los habitantes populares son los principales agentes de la urbanización. La autoconstrucción ha posibilitado la irrupción de nuevas formas de ciudadanía en las cuales los pobres han generado nuevos espacios de participación cívica que los han convertido en legítimos portadores de derechos. ¿Qué ocurre, sin embargo, cuando los agentes de la autoconstrucción son inmigrantes demandando no solo el derecho a la vivienda, sino también su reconocimiento como sujetos de derechos en un Estado-nación que no los considera ciudadanos? Este artículo discute dicha pregunta examinando etnográficamente el caso del campamento Nueva Esperanza, asentamiento autoconstruido por inmigrantes de origen latinoamericano en la comuna de Colina, Santiago. Concluimos que, para constituirse como ciudadanos, los extranjeros formulan un tipo "urbano" de ciudadanía, en donde el acto de residir en la ciudad los convierte en legítimos portadores de derechos. En dicho proceso, estos residentes construyen narrativas éticas y políticas a través de las cuales significan sus deseos de incorporación y pertenencia a la comunidad política nacional.

Palabras clave: ciudadanía urbana, migración, autoconstrucción, asentamientos informales.

\begin{abstract}
In several metropolises of the so-called Global South, the claim for the right to live in the city has resulted in widespread processes of "autoconstruction", a concept that alludes to a mode of producing the urban peripheries in which poor residents are the main agents of urbanization. Autoconstruction has made possible the emergence of new forms of citizenship in which the urban poor have generated novel spaces for civic participation through which they have turned into legitimate rights-bearers. What happens, however, when the agents of autoconstruction are immigrants demanding not only the right to housing, but also their recognition as subjects of rights in a nation-state that does not consider them citizens? This article discusses
\end{abstract}


that question by ethnographically examining the case of Nueva Esperanza squatter settlement, an autoconstructed neighborhood in Colina, Santiago in which most of its residents are foreigners. We conclude that, to constitute themselves as citizens, immigrants formulate an "urban" type of citizenship in which the act of residing in the city turn them into rights-bearers. In such a process, these residents build ethical and political narratives through which they make sense of their desires for incorporation and belonging to the national political community.

Keywords: urban citizenship, migration, autoconstruction, squatter settlements.

Recibido: 7 mayo 2019. Aceptado: 13 marzo 2020 


\section{Introducción}

Chile ha experimentado un aumento sostenido de inmigración latinoamericana desde la década de los noventa hasta la actualidad. Durante los últimos años, a la migración de países fronterizos como Perú, Bolivia y Argentina se han sumado migraciones de países de mayor lejanía geográfica como Colombia, República Dominicana, Venezuela y Haití. Según datos del Instituto Nacional de Estadísticas, INE (2020), en el país viven cerca de 1499522 personas de origen extranjero, equivalentes a cerca del $8 \%$ de la población, muy por sobre el $0,81 \%$ registrado en 1992.

En un contexto en el que la presencia de inmigrantes ha crecido de manera considerable, las investigaciones sobre el fenómeno han puesto su atención en dos grandes dimensiones de análisis. Por una parte, existe un importante grupo de trabajos que se ha enfocado en examinar los procesos de discriminación, exclusión y racialización experimentados por inmigrantes latinoamericanos (Pavez, 2016; Sheehan, 2018; Stefoni y Fernández, 2011; Tijoux, 2013; Tijoux y Palominos, 2015). Por otra parte, están aquellos estudios que, analizando las prácticas sociales de los inmigrantes y los procesos de diversificación cultural que traen consigo, reflexionan sobre los efectos de la migración en aspectos como la identidad o las concepciones tradicionales de nación, ciudadanía y territorio (A. Garcés, 2012; Guizardi y Garcés, 2013; Imilan, 2014; Márquez, 2014; Stefoni y Stang, 2017; Garcés y Maureira, 2018). Aunque con algunas pocas excepciones (Margarit y Bijit, 2014; Margarit y Galaz, 2018), las prácticas residenciales de los inmigrantes y sus implicancias ético-políticas han sido, sin embargo, menos exploradas. En este marco, en el presente artículo nos preguntamos por las maneras en que la experiencia de poblar informalmente la ciudad, fruto de la exclusión urbana hacia los inmigrantes latinoamericanos (López-Morales et al., 2018; Sheehan, 2018), puede dar paso a la configuración de nuevos tipos de ciudadanía basados en la residencia en donde estos habitantes reconfiguran sus prácticas e imaginarios políticos en tanto sujetos de derecho.

¿Por qué preguntarse por la ciudadanía desde una perspectiva que rescata la producción de los inmigrantes de sus espacios residenciales? Una viñeta etnográfica nos permite abordar dicha pregunta. Angélica, una dominicana de poco más de cuarenta años, residía en los márgenes del área metropolitana del Gran Santiago, específicamente en el campamento Nueva Esperanza de Colina. Como gran parte de sus compatriotas que viven en ese asentamiento, Angélica llegó a Chile a inicios de la década pasada a trabajar como asesora del hogar. La poca estabilidad del servicio doméstico la forzó a ocuparse en una serie de trabajos precarizados como parquímetro humano en la vía pública o vendedora callejera. Más que como "inmigrante”, ella, al igual que muchos de sus vecinos, prefiere referirse a sí misma como "extranjera", tal vez como un modo de alejarse del carácter estigmatizador frecuentemente asociado al primer término. ${ }^{1}$ Luego de vivir casi siete años como arrendataria, decidió mudarse al campamento en 2017. "¿Por qué crees que tienes derechos en este país?”, le preguntamos en una de nuestras primeras visitas a Nueva Esperanza. Ella, tajantemente, respondió:

1 Para quienes colaboraron en esta investigación, la categoría "extranjero" resultaba socialmente distinta a la de "inmigrante", principalmente por las improntas racistas e identitarias que se le han impuesto a la segunda. En este artículo, decidimos usar la noción de "extranjero", ya que dicho término era el que usaban mayormente nuestros interlocutores al momento de referirse a sí mismos. Reservaremos, sin embargo, el uso del concepto "inmigrantes" para aludir a las investigaciones que han abordado la materia, en razón de que esta ha sido la noción predominante con que se ha categorizado a estos sujetos en las ciencias sociales. 
Nosotros [los extranjeros] tenemos más derechos porque pagamos impuestos. $Y$ hay chilenos que viven aqui y que no pagan impuestos, ¿entiende? Aqui hay chilenos que viven de lo que le dan ¿entiende? [...]. Hay chilenos que son muy vagos aqui, que no les gusta trabajar. Entonces nosotros venimos con un propósito aqui, a trabajar [...]. Yo ahora no estoy trabajando, yo estoy incómoda, porque no estoy trabajando. No estoy trabajando ahora, pero puedo trabajar mañana, ¿entiende?

La opinión de Angélica da luces importantes sobre el modo en que estos autoconstructores conceptualizan la ciudadanía y los derechos. Su apreciación, en efecto, nos permite explorar los elementos definitorios de un tipo de imaginación política fundada en la idea de que aquellos que contribuyen más a la sociedad merecen más derechos que otros. La autoconstrucción de viviendas y campamentos es, precisamente, una de las formas por las que nuestros interlocutores afirmaban materializar su contribución a la sociedad receptora legitimando, con ello, su ejercicio de derechos. En este artículo sostenemos que el reconocimiento de la autoconstrucción como una práctica política posibilita la emergencia de una ciudadanía urbana. En tanto ciudadanía "localizada" que se desancla de las formas de reconocimiento jurídico basados en la membresía nacional (Sassen, 2002), la ciudadanía urbana que aquí discutimos permite que los inmigrantes se constituyan como sujetos capaces de reivindicar derechos al Estado, independientemente de su país de origen o condición migratoria.

Para definir esta ciudadanía urbana, partiremos caracterizando el proceso migratorio en Santiago de Chile, enfocándonos en la experiencia urbano-residencial de los inmigrantes. Luego, describiremos etnográficamente las prácticas de autoconstrucción a través de las cuales los habitantes como Angélica han producido material y simbólicamente sus espacios residenciales. Mediante el concepto de "ciudadanía urbana" (Holston y Appadurai, 1996; Holston, 2008; Pérez, 2017), discutiremos, posteriormente, la relación entre ciudadanía y habitar, afirmando que el acto de autoconstruir un espacio residencial se vuelve el principal criterio de membresía política utilizado por los inmigrantes. Finalmente, concluimos que el proceso de autoconstrucción de los inmigrantes tiene dos elementos definitorios: 1) a diferencia de las movilizaciones urbanas que emergieron en décadas anteriores, la aparición de campamentos culturalmente diversos resultan de prácticas de producción de espacio individuales, más que colectivas, que se ven permeadas por una racionalidad de mercado; 2) para ser reconocidos como ciudadanos urbanos, los extranjeros articulan narrativas éticas y políticas sobre sus condiciones de exclusión por las que significan sus deseos de incorporación y pertenencia a la comunidad política nacional. En estas narrativas, sostenemos, se ancla un entendimiento paradójico de los derechos: aunque los extranjeros imaginan los derechos como una prerrogativa universal de cualquier individuo en tanto ser humano, al mismo tiempo articulan narrativas basadas en categorías morales (esfuerzo, decencia, sacrificio, etc.) a través de las cuales niegan la capacidad de ejercer derechos a otros residentes del campamento.

Este artículo resulta de un proyecto de investigación etnográfico en el que aplicamos 30 entrevistas en profundidad y observación participante al interior del mismo campamento durante ocho meses, específicamente desde agosto de 2017 a marzo 2018. Los entrevistados fueron seleccionados siguiendo un tipo de muestreo no probabilístico (técnica de "bola de nieve"). El material textual proveniente de entrevistas y notas de campo fue analizado mediante la "teoría fundamentada" (Glaser y Strauss, 2017), proceso interpretativo de carácter inductivo en el cual los datos empíricos organizan y determinan las reflexiones teóricas aquí expuestas. Para proteger la identidad de nuestros interlocutores, todos los nombres propios que aquí aparecen son seudónimos. 


\section{Experiencia residencial de los inmigrantes en Santiago}

Para 2019, 789412 migrantes residían en la región Metropolitana, equivalentes a cerca del $11 \%$ de la población regional (INE, 2019). De ellos, destacaban principalmente personas provenientes de Venezuela (26,8\%), Perú $(21,4 \%)$ y Haití $(13,9 \%)$. En la experiencia de los inmigrantes avecindados en Santiago, se han conjugado procesos de inclusión/exclusión que están directamente relacionados con el lugar ocupado en la ciudad y las prácticas urbanas que estos despliegan. Así lo han señalado, por ejemplo, estudios sobre cómo el comercio inmigrante y la búsqueda de empleo en la ciudad posibilitan tanto un anclaje en espacios específicos como la creación de vínculos que les permiten a los inmigrantes apropiarse de ciertas áreas de la ciudad (A. Garcés, 2012; Imilan, 2014; Stefoni, 2016). La experiencia urbana de los inmigrantes, al mismo tiempo, se construye a partir de la visibilidad a sus expresiones culturales; una visibilidad que muchas veces es negada y usada como justificación para articular discursos racistas y criminalizadores que guardan la exigencia asimilacionista de "que se deje de ser aquello que lo diferencia para transformarse así en uno más del grupo” (Stefoni, 2013, p. 59).

En un contexto donde los inmigrantes están sometidos a discursos y prácticas de rechazo y exclusión por parte de la sociedad receptora, la experiencia residencial y habitacional es una de las más significativas en la dimensión urbana de los procesos migratorios. Como señala Vacotti (2017), el "adentro" de sus barrios muchas veces sirve como un contrapunto al rechazo que deben enfrentar en el espacio público. La residencia, al mismo tiempo, sirve para la articulación de redes locales con connacionales, la búsqueda de empleo y como un enclave para la afirmación de las especificidades culturales (Luque, 2004; Margarit y Bijit, 2014; Contreras et al., 2015).

En el caso de Santiago, hacia la década de los noventa y principios de los dos mil, los inmigrantes comenzaron a poblar ciertas áreas deprimidas en términos habitacionales y comerciales, particularmente en la zona norte del casco histórico de Santiago y en algunas contiguas como Recoleta e Independencia. Tras este fenómeno, vinieron a ocupar una zona residencial que se encontraba prácticamente en abandono luego del desplazamiento de habitantes de origen nacional hacia nuevas comunas y zonas residenciales en la zona suroriente de la región Metropolitana de Santiago (Luque, 2004). Con la llegada de los inmigrantes, entonces, se produjo una reactivación comercial y habitacional en dichas zonas, permitiendo la emergencia de barrios con alta concentración de población extranjera.

Hacia finales de los 2000s, la distribución por nacionalidades entre inmigrantes parecía no tener un patrón específico, mostrando cierta aleatoriedad residencial y un rechazo a los "nichos étnicos" (Schiappacasse, 2008). Trabajos posteriores, sin embargo, discuten esta tesis (Margarit y Bijit, 2014; Márquez, 2014; Contreras et al., 2015). El patrón residencial de los migrantes, sostienen directa o indirectamente dichos estudios, no resulta azaroso, estando estrictamente relacionado con factores como la condición socioeconómica, el país de procedencia y el género a partir de los cuales se articulan prácticas discriminatorias en el acceso a la vivienda.

De acuerdo al Censo 2017, los inmigrantes se establecen en las áreas centrales y pericentrales de la ciudad, particularmente en comunas como Santiago (28\%), Estación Central (17\%) y Recoleta (16\%). Si bien dicha tendencia parece haberse mantenido, ${ }^{2}$ en los últimos años la

2 En efecto, en la comuna de Santiago residían 212037 extranjeros hacia 2019 (INE, 2019), muy por sobre los 84094 que contabilizó el Censo 2017 (ver más en Atisba, 2018). 
elección residencial de los migrantes ha mostrado nuevos visos. En primer término, ha existido una revitalización del mercado inmobiliario en zonas céntricas que antiguamente no tenían fines habitacionales, lo que ha llevado a una ampliación del mercado formal e informal de la vivienda (Contreras et al., 2015; Sheehan, 2018). Dicho fenómeno ha recrudecido las condiciones habitacionales de la población inmigrante de origen latinoamericano, limitando su acceso, en el caso de la población de menores ingresos, casi exclusivamente a viviendas tugurizadas y con escasas condiciones de habitabilidad (Margarit y Bijit, 2014; Contreras et al., 2015). Delia Curahua, de la Asociación de Mujeres Inmigrantes Warmipura, señala:

[Los lugares que habitan las personas inmigrantes] son precarios, deficientes en instalaciones eléctricas, sin permiso incluso para funcionar como espacios habitables, pero cuyo precio de costo fluctúa entre los 60 mil y 200 mil pesos [US $\$ 92$ y US\$307 respectivamente] dependiendo de si son espacios céntricos (Santiago, Independencia, Recoleta) carentes de servicios higiénicos adecuados, con pasajes angostos, donde las familias cocinan sus alimentos y donde necesariamente al pasar parte de sus vidas en convivencia se generan los roces entre ellos y problemas graves consecuencia del hacinamiento (Curahua, 2013, p. 44).

En segundo término, y asociado con esto último, se ha constatado que en la elección residencial también cobra interés las potencialidades territoriales, laborales y sociales del lugar donde se vive (Margarit y Bijit, 2014). Esto explica que, ante la excesiva demanda de vivienda en las áreas centrales y sus abusivas condiciones, se observen desplazamientos hacia comunas alejadas del centro de la ciudad, pero que también concentran alta actividad comercial y laboral. Es el caso de las comunas de la zona norte de la región Metropolitana como Quilicura, Lampa, Til Til y Colina. Siendo una comuna de la "extraperiferia" (Jirón y Mansilla, 2014, p. 15), Colina destaca por ser un distrito sometido a importantes transformaciones inmobiliarias durante las últimas décadas, asociadas a la construcción de viviendas de lujo para las clases más acomodadas de la región Metropolitana. Ello ha llevado a un aumento de las actividades laborales asociadas al servicio doméstico y la construcción, cuestión que resulta clave para entender las opciones de localización de los inmigrantes. En efecto, el Censo 2017 muestra que de las 146 207 personas que residen en Colina, 2627 son de origen extranjero, las que provienen principalmente de Haití (19,6\%) y República Dominicana (18,2\%).

No resulta extraño que, ante los obstáculos de acceso a la vivienda y las condiciones abusivas de este mercado, la necesidad de los inmigrantes latinoamericanos de establecerse territorialmente esté dando paso a un aumento significativo de la presencia de estos en campamentos o asentamientos informales (López-Morales et al., 2018). Las estadísticas, de hecho, así lo avalan. Si en 2015, solo un 6\% de las familias de campamentos a nivel nacional eran de origen extranjero (CIS-TECHO, 2015), en 2021 esa cifra aumentó al 30,58\% (CES-TECHO, 2021). No obstante, más allá de los estudios que caracterizan la habitación informal de los inmigrantes o que constatan las razones de por qué ellos están llegando a los campamentos (Vicuña y Rojas, 2015; Rojas y Silva, 2016; López-Morales et al., 2018), los alcances ético-políticos del aumento de familias inmigrantes en campamentos han sido escasamente explorados. Más aún, poco se ha dicho sobre cómo los moradores extranjeros se forman como ciudadanos a partir de las prácticas de autoconstrucción por las que dan vida a sus espacios residenciales. 


\section{Autoconstrucción y ciudadanía urbana}

Desde hace más de cinco décadas, diversos autores han destacado la íntima relación entre la falta de viviendas para los sectores populares y la emergencia de movilizaciones por derechos de ciudadanía en ciudades latinoamericanas (Mangin, 1967; Turner, 1968; Castells, 1983; Holston, 2008; Murphy, 2015; Caldeira, 2017). El reclamo por el derecho a vivir en la ciudad se ha materializado en extensos procesos de autoconstrucción, término que alude al fenómeno según el cual los residentes populares construyen ellos mismos sus espacios residenciales (Holston, 2008; Caldeira, 2015, 2017). Para Teresa Caldeira (2015), la autoconstrucción forma parte de lo que llama "urbanización periférica", a saber, una modalidad de producir el espacio urbano que tiene tres características. Primero, ser un proceso prolongado en el tiempo en el que los autoconstructores están constantemente redefiniendo, rehaciendo y remodelando sus espacios residenciales. Segundo, gatillar un modo específico de relacionamiento con las lógicas oficiales del Estado y el mercado. Si bien operan en la informalidad, señala Caldeira, los autoconstructores lidian constantemente con problemas de legalización, regulación y especulación, al tiempo que consumen mercancías para erigir sus viviendas. Tercero, generar nuevos tipos de ciudadanía y circuitos de contestación a partir de los cuales los autoconstructores se constituyen como sujetos políticos capaces de reivindicar legítimamente derechos a los Estados.

En el caso de Chile, el fenómeno de la autoconstrucción ha sido mayormente examinado en relación al llamado "movimiento de pobladores". En dicho movimiento, miles de familias sin casa conquistaron su derecho a la vivienda ocupando masivamente predios urbanos en donde, con posterioridad a la toma de terrenos, erigían campamentos (Espinoza, 1988; M. Garcés, 2002; Murphy, 2015; Angelcos y Pérez, 2017). El movimiento de pobladores fue particularmente intenso entre mediados de los ańos cincuenta y 1973 cuando, en un contexto de politización creciente, partidos de izquierda y centroizquierda empezaron a ver en los pobres urbanos una fuerza importante de transformación social (Castells, 1983; Angelcos y Pérez, 2017). La construcción del poblador como un sujeto potencialmente revolucionario se consolidó durante el gobierno de Salvador Allende (1970-1973), período en el cual los partidos de la Unidad Popular dirigieron las ocupaciones de suelo urbano para llevar a cabo sus agendas partidistas a nivel territorial. Esto llevó a la aparición de más de 300 campamentos en Santiago (Cofré, 2011), la mayoría de los cuales estaba política e ideológicamente apoyado por un partido político específico. Así, para 1973 los campamentos de Santiago congregaban a más de 83000 familias, lo que equivalía a 16,3\% de la población de Santiago (Santa María, 1973).

El número de familias viviendo en campamentos ha crecido exponencialmente en los últimos dos años, específicamente, de 47050 en 2019 a 81643 en 2021 (CES-TECHO, 2021). En el caso de la región Metropolitana, tal proceso implicó un aumento de 5991 a 19444 familias en el mismo período. Los campamentos de la actualidad, sin embargo, presentan algunas diferencias con aquellos formados en el marco del movimiento de pobladores. Dos elementos dan cuenta de ello: primero, la irrupción de ocupaciones ilegales no necesariamente deriva de la acción concertada de pobladores altamente politizados, quienes, organizados por partidos políticos de izquierda en disputa por el poder político, participan masivamente de movimientos sociales urbanos. Aunque en el contexto del estallido social de 2019 emergieron algunos campamentos cuyos residentes articularon un claro lenguaje reivindicativo -por ejemplo, el campamento Dignidad en La Florida y la toma Macarena Valdés en Cerro Navia-, los niveles de radicalidad y organización de estos asentamientos son más bien excepcionales. Así, en el Chile neoliberal, las familias pobres parecen concebir los campamentos como una estrategia 
individual para acceder a predios urbanos bien localizados y, eventualmente, transformarse en propietarios de una vivienda subsidiada (Brain et al., 2010). ${ }^{3}$ Segundo, los campamentos del presente son, en comparación con los del pasado, más diversos en términos culturales debido a la llegada sistemática de inmigrantes en la última década, fenómeno que ha incidido en la progresiva "etnificación" ${ }^{4}$ del espacio urbano (Imilan et al., 2014). Como hemos mencionado, algunos estudios indican que los inmigrantes están sistemáticamente expuestos a prácticas de discriminación cuando tratan de acceder al mercado formal de la vivienda (Contreras et al., 2015; Sheehan, 2018). Ello explicaría el aumento importante de inmigrantes residiendo en asentamientos informales desde donde articulan demandas por ser reconocidos como sujetos de derechos. ¿Por qué entender tales reclamos como reivindicaciones por derechos de ciudadanía?

Desde la antropología, el concepto de ciudadanía ha sido definido como un sistema regulatorio a través del cual las comunidades políticas administran sus diferencias internas con el fin de consolidar sus proyectos identitarios (Kipnis, 2004; Postero, 2007; Holston, 2008). Todas las formulaciones de ciudadanía operan sobre la base de una lógica de inclusión/exclusión materializada en criterios de membresía política que delimitan el tipo de derechos que pueden ejercer quienes son reconocidos como ciudadanos (Kipnis, 2004). La ciudadanía, señala Nancy Postero (2007), refiere por tanto a una relación entre individuos o grupos y el Estado; relación que está enmarcada por cómo las sociedades administran el problema de la pertenencia a la comunidad política.

En la formulación liberal-moderna de ciudadanía, la nacionalidad ha sido entendida como el criterio primordial para definir quién pertenece a la sociedad. Alimentada y fortalecida a través de la emergencia del Estado moderno en Europa (Spruyt, 1994), este tipo de ciudadanía asumió la forma de un "estatus" (Marshall, 1977; Walzer, 1989) para los miembros de la nación, la que comenzó a ser concebida como una comunidad de intereses y valores compartidos (Anderson, 1991). Así, quienes pertenecen a una nación y poseen el estatus de ciudadano son formalmente iguales en dignidad y derechos. El que los miembros de una nación sean reconocidos como ciudadanos no implica, claro está, una igualdad sustantiva entre ellos. La desigualdad social, en efecto, ha sido una constante en las sociedades que han asumido este régimen de ciudadanía. El principio de igualdad formal que defiende la ciudadanía moderna, más bien, remite a la idea de que los ciudadanos tienen igual capacidad de ejercer los mismos derechos y de pedir protección al mismo corpus legal (Walzer, 1989).

Desde la segunda década del siglo XX, el modelo nacional-moderno de ciudadanía ha sido fuertemente cuestionado por su incapacidad de asegurar de manera efectiva la participación de grupos excluidos en la esfera política. Las aproximaciones críticas han sostenido que la promoción de igualdad formal entre los ciudadanos, el principio fundante de la ciudadanía moderna, ha llevado a la subordinación de la diferencia social -ya sea en términos de raza, género, clase, etcétera- a las estructuras culturales dominantes (Young, 1989; Taylor, 1992; Kymlicka, 1995; Benhabib, 2002). Una de las perspectivas críticas más reconocidas, la "ciudadanía diferenciada” de Iris Young (1989), propugnaba la creación de un sistema de asignación de más y mejores derechos a aquellos que han vivido en condiciones de exclusión estructural y sistemática.

3 Esto no implica negar la posibilidad de que, ante una creciente rearticulación de los movimientos sociales, los campamentos vuelvan a tomar un rol protagónico en la disputa por el poder.

4 Imilán, Garcés y Margarit (2014, p. 26) entienden por "etnificación” del espacio urbano aquel proceso en el que los inmigrantes, al desplegar prácticas cotidianas fuera de sus países de nacimiento, actualizan recursos y capitales sobre la base de una pertenencia a un origen compartido. 
La pregunta que subyace a propuestas como la "ciudadanía diferenciada" de Young es de qué manera colectivos excluidos pueden demandar legítimamente derechos cuando, para el modelo nacional de ciudadanía, ellos carecen de estos. En este artículo, afirmamos que el proceso de autoconstrucción de la ciudad llevado a cabo por los migrantes da importantes luces al respecto. Siguiendo debates recientes en antropología sobre autoconstrucción y producción de espacios periféricos (Caldeira, 2015, 2017), sostenemos que dichas prácticas de construcción de ciudad posibilitan la articulación de narrativas sobre derechos que superan las limitaciones propias del proyecto nacional-moderno de ciudadanía, dando pie a un modelo "urbano" de ciudadanía.

El concepto de "ciudadanía urbana" ha emergido con fuerza entre antropólogos y cientistas sociales interesados en examinar las potencialidades políticas de las ciudades en un contexto de urbanización planetaria (p.e., Holston y Appadurai, 1996; Bauböck, 2003; Holston, 2008, 2019; Lebuhn, 2013; Blokland et al., 2015; Pérez, 2017). Tal idea ha sido empleada para examinar, por ejemplo, el ejercicio de derechos cuando se transforman las fronteras nacionales (Lebuhn, 2013) o la irrupción de formas de autogobierno orientadas a la creación de una ciudadanía emancipada de los imperativos de la ciudadanía nacional. Aquí, en cambio, usaremos el concepto en cuestión para comprender cómo se legitima la capacidad de ejercer derechos por el hecho mismo de habitar en la ciudad. En ese sentido, siguiendo a James Holston (2008, 2019; cf. Pérez, 2017), concebimos la ciudadanía urbana como aquella formulación de ciudadanía que tiene tres características: a) considerar la ciudad, más que la nación, como la comunidad política primordial; b) entender el habitar, más que la nacionalidad, como el criterio central de membresía política, y; c) pensar los derechos reivindicados como derechos a la ciudad. Todos los regímenes de ciudadanía, señala Holston (2008), se gestan como ensamblajes coyunturales e históricos en los que coexisten distintos modelos de distribución de derechos, sean estos forjados en una formulación "nacional", "urbana", "diferenciada", etcétera. En ese sentido, la ciudadanía urbana, más que como régimen que reemplaza la ciudadanía nacional, debe concebirse como una práctica que la complejiza y la cuestiona, aun cuando el modelo nacional-moderno siga predominando.

La residencia como criterio general de membresía de la ciudadanía urbana debe, sin embargo, tomarse con precaución. Para Henri Lefebvre (1996), el habitar se materializa a través de acciones transformativas tendientes a profundizar los grados de igualdad y justicia social de nuestras ciudades. Ello implica que, como categoría abstracta, el habitar es vacío y carente de sentido si no se despliega en la praxis. Por tanto, la ciudadanía urbana, al basarse en el habitar, debiera entenderse menos como un estatus que como una reivindicación o claim (cf. Das, 2011). Siendo más una práctica que un estatus legal fijo, ${ }^{5}$ la ciudadanía urbana descansa en la demanda constante de derechos, en los que los ciudadanos urbanos buscan no solo la ampliación de los ya existentes, sino también la creación de nuevos derechos. Así pensada, esta formulación urbana de la ciudadanía, en tanto ensamblaje "insurgente" (Holston 2008) que emerge dentro de la ciudadanía nacional, podría asegurar lo que el proyecto moderno fue incapaz de promover: la inclusión y representación de los grupos excluidos sin importar su pertenencia formal al Estado-nación.

5 Para profundizar en las diferencias entre la ciudadanía entendida como "práctica” o como "estatus", ver la interesante comparación de Walzer (1989) entre los tipos de ciudadanía "republicana” e "imperial/ liberal". 


\section{Nueva Esperanza: “vivir bien” en un campamento de "extranjeros"}

El campamento Nueva Esperanza fue formado en 2016, mediante la ocupación de un predio de aproximadamente una hectárea, en las inmediaciones del Centro Penitenciario Colina II y el Cementerio Municipal de la comuna. Ubicado a menos de 500 metros hacia el oriente de la avenida General San Martín, la principal arteria de transporte público de Colina, Nueva Esperanza es un asentamiento de fácil acceso. Tal vez por ello su emergencia -iniciada por chilenos y extranjeros, aunque consolidada fundamentalmente por los segundos- fue rápida. Para abril de 2018, este campamento congregaba a cerca de 80 familias distribuidas, en palabras de una residente dominicana, en "seis razas": colombiana, peruana, boliviana, dominicana, haitiana y chilena. Aunque dominicanos, haitianos y colombianos parecían predominar en términos numéricos, resultaba difícil estimar el número exacto de moradores y su distribución por nacionalidad, puesto que la llegada de residentes continuaba.

Nueva Esperanza, por ello, ejemplifica de manera sugerente el carácter dinámico y progresivamente diversificado de los campamentos en Chile, lo que se expresa en la presencia creciente de elementos "foráneos" como banderas (Figura 1), productos extranjeros, comidas y celebraciones. Igualmente, la residencia de población extranjera en Nueva Esperanza ha resultado en la aparición de modalidades específicas de cuidado y mantención de las viviendas que, inspirados en la idea de "vivir bien", nos permitirán, más adelante, analizar la emergencia de una ciudadanía urbana entre inmigrantes latinoamericanos.

La mayor parte de los residentes de Nueva Esperanza con quienes pudimos dialogar habían llegado al campamento a través de un familiar o conocido. Antes de arribar a la ocupación, habitaban en viviendas sociales circundantes al terreno donde se emplaza el campamento, en condiciones abusivas de arriendo y subarriendo y la amenaza constante de desalojos y embargos. Un buen porcentaje de ellos llegó a Colina buscando oportunidades laborales en el sector de servicios, donde se ha generado un importante polo económico a partir de la instalación de barrios para residentes de altos ingresos en Chicureo y otras urbanizaciones aledańas. Así, gran parte de los residentes de Nueva Esperanza se empleaban en labores como el servicio doméstico y el cuidado de niños, en el caso de las mujeres; y trabajos de seguridad, construcción y jardinería, en el caso de los hombres. Sumado a ello, existía un considerable número de moradores que trabajaba en el sector informal, sobre todo en la venta de alimentos, las ferias libres o en negocios instalados al interior de sus casas. En estos últimos, pudimos observar una diversidad de servicios, que iban desde la venta de comida hasta cortes de pelo, manicure, entre otros. Este tipo de autoempleo era desarrollado mayoritariamente por extranjeros que se encontraban en situación irregular, puesto que "estar sin papeles" dificultaba de manera sustantiva las posibilidades de acceso a empleos formales. Si bien la mayoría de los residentes de Nueva Esperanza poseía una situación regular en el país -algunos tenían residencia definitiva-, un importante grupo de dominicanos se encontraba en situación irregular. Esto, principalmente debido al restrictivo visado para inmigrantes del país, que se fijó en el ańo 2012 durante el primer gobierno de Sebastián Piñera, que ha fomentado, desde entonces, los ingresos irregulares mediante la frontera terrestre (Ortiz, 2019). Del total de nuestros entrevistados de origen dominicano, al menos cinco se encontraban en situación irregular al momento de conocerlos. 


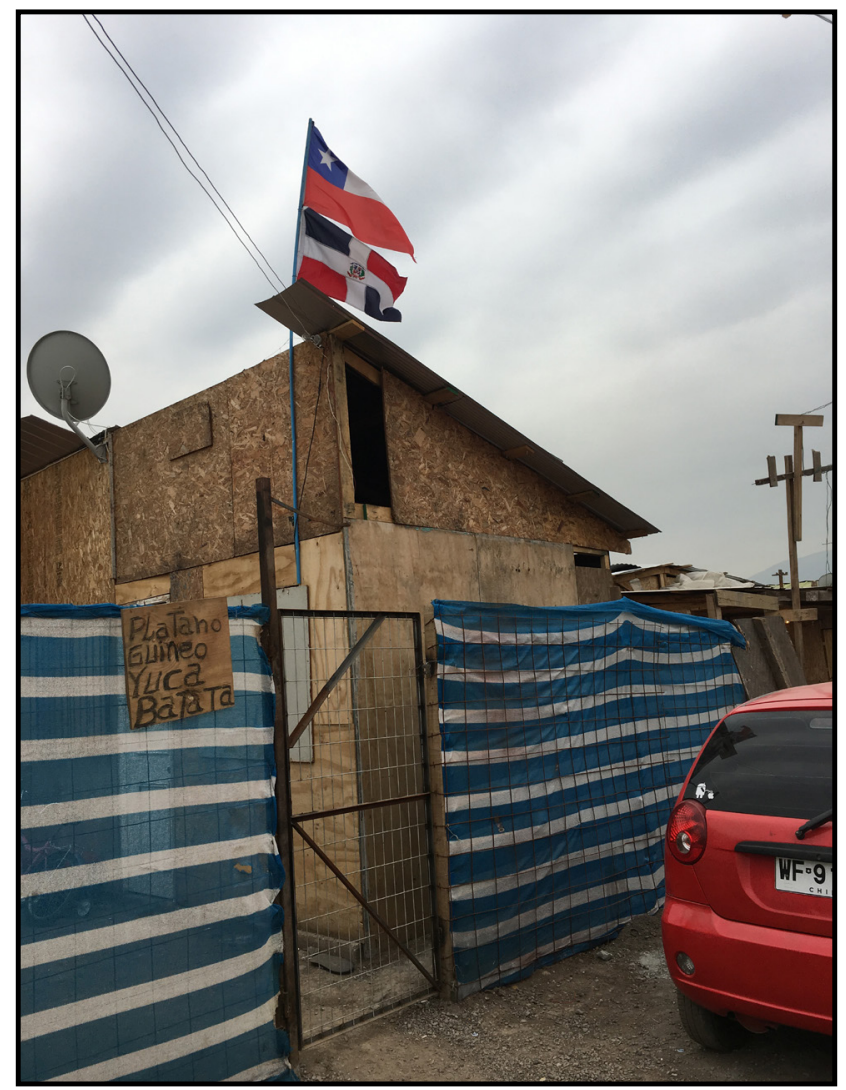

Figura 1. Bandera dominicana junto a la chilena en Nueva Esperanza. Fuente: Fotografía de Miguel Pérez.

Las viviendas del campamento eran similares en términos estructurales. Construidas con planchas OSB (paneles de madera aglomerada) y techos de zinc, el conjunto de casas que formaban Nueva Esperanza presentaba similitudes evidentes en su materialidad estructural. No obstante, las diferencias estéticas entre las viviendas erigidas por chilenos y por inmigrantes se volvían bastante notorias en las terminaciones. Mientras las familias chilenas tendían a construir sus espacios residenciales de manera mucho más rudimentaria y poco sofisticada, las familias extranjeras parecían mucho más dedicadas y comprometidas, cuestión que se volvía patente al comparar los materiales usados en puertas, pisos, rejas, etcétera. La Figura 2 muestra a un residente colombiano preparando los materiales para la autoconstrucción de su vivienda. Mientras la mayoría de sus vecinos chilenos presenta rejas hechas de malla de polietileno, restos de madera y planchas de zinc, él ha levantado una reja sólida con alambres de púas para la protección de su casa.

¿Cómo explicar esta diferencia? Según el relato de nuestros entrevistados, los extranjeros invertían más dinero en adornar su casa que los chilenos, hecho que los hacía ser identificados como los "ricos" del campamento. Sin embargo, más allá de la veracidad empírica de este juicio, sostenemos que las diferencias estéticas observables en las casas de los residentes foráneos revelan el modo en que estos se conciben a sí mismos como ciudadanos. El especial cuidado de la vivienda que demostraban estos residentes expone, en ese sentido, las formas en que ellos significaban cotidianamente su existencia como sujetos de derechos. Un ejemplo etnográfico nos ayudará a iluminar este punto. 


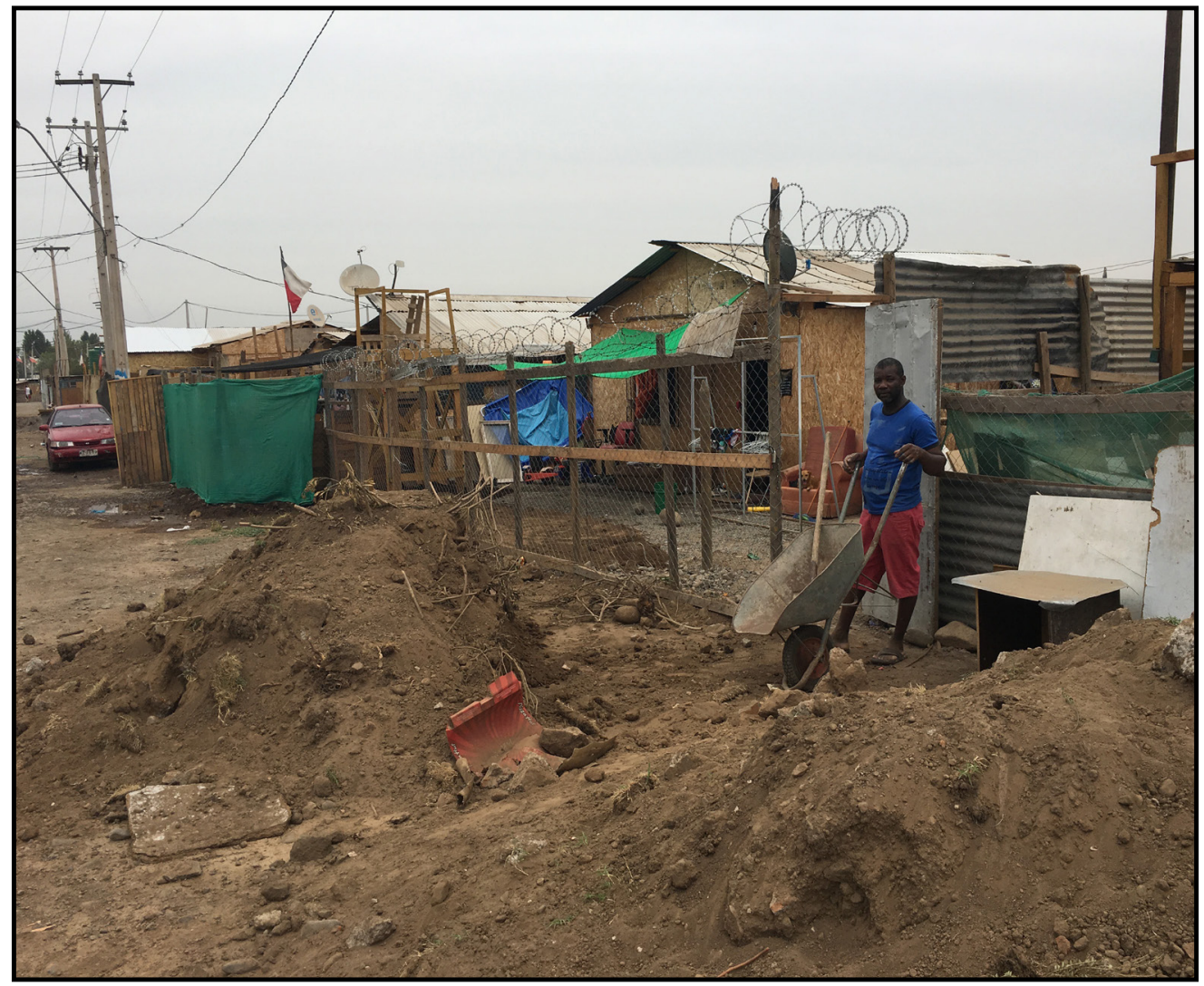

Figura 2. Autoconstrucción inmigrante en Nueva Esperanza. Fuente: Fotografía de Miguel Pérez.

Adrián, un dominicano cercano a los treinta años, llegó a Chile en 2013. Cuando lo conocimos, se desempeñaba laboralmente como jardinero en Chicureo. Ingresó al país como indocumentado desde Bolivia para vivir con sus primos, quienes ya se habían instalado en Colina algunos años antes. "Entré ilegalmente, pero gracias a Dios ya tengo la cédula [de identidad]", afirmó en el primer encuentro que tuvimos con él. En 2017, junto a su pareja -una dominicana que Adrián conoció en Colina- decidieron mudarse al campamento ya que el propietario de la vivienda que arrendaban amenazó con subir el precio del alquiler. En esas circunstancias, tanto él como su pareja vieron en Nueva Esperanza la posibilidad de seguir viviendo en Colina sin la presión de enfrentarse a una futura alza.

Para Adrián, la llegada al campamento no fue gratuita, ya que implicó el desembolso de 200 000 pesos (alrededor de 330 dólares). En nuestro trabajo de campo escuchamos historias similares en varias ocasiones. En todas ellas, los extranjeros aseguraron haberles "comprado" un terreno a chilenos que, en las etapas iniciales del campamento, habían loteado el predio de manera completamente irregular. Las vivencias de Adrián y de muchos de nuestros interlocutores develan, en ese sentido, dos aspectos importantes: primero, la racionalidad de mercado operando en la emergencia de los asentamientos informales, hecho expresado en que los ocupantes extranjeros literalmente pagaron por su derecho a vivir en el campamento; segundo, el carácter discriminatorio de este mercado informal de la vivienda puesto que, según lo observado empíricamente, los inmigrantes eran los únicos forzados a gastar dinero por el derecho a autoconstruir. Sin embargo, los extranjeros como Adrián desafiaban ese tipo 
de discriminaciones generando un conjunto de juicios éticos y estéticos a través de los cuales se constituían como individuos legítimamente dotados de derechos. El hecho de invertir más dinero en la construcción de viviendas era, en este marco, imaginado como una práctica propia de ciudadanos virtuosos, trabajadores y sacrificados. En sus palabras:

\section{Los chilenos viven como los animales [...]; los inmigrantes como los colombianos, los} haitianos, los dominicanos, los peruanos, todos tienen su buena casita [pero los chilenos] son viciosos; yo voy a tener seis años aqui y me levanto a las seis de la mañana para ir a trabajar y ellos [los chilenos] no se van a levantar. Entonces, por eso uno puede comer bien, vivir bien. [Los chilenos] no trabajan... Algunos trabajan, algunos no, algunos usted los ve que se levantan a las diez a pedir.

Entrevistador: ¿Qué quiere decir "viven como animales" para usted?

Animales es que... el animal come lo que encuentra, ellos hallan un pájaro y se lo comen y no les importa vivir en la cochinada. A uno [en cambio] le gusta vivir bien.

En el interior de la casa de Adrián sobresalían las cerámicas con que había acondicionado su piso. Destacaban, también, la cocina estilo americano que él había construido y una serie de artefactos que lucía orgullosamente, como un televisor LCD de 40 pulgadas y una antena de televisión satelital. Según nos contó, autoconstruir su vivienda había implicado el gasto de más de cuatro millones de pesos (aproximadamente 6500 dólares), cifra que, aunque elevada, no era muy distinta de lo que otros extranjeros aseguraban haber gastado para "vivir bien".

La autoconstrucción involucra una temporalidad específica organizada por la constante renovación del espacio construido (Caldeira, 2015, 2017). Las casas y los barrios crecen gradualmente en un proceso de largo alcance durante el cual los residentes mejoran sus espacios residenciales continuamente. En el contexto del campamento, el "vivir bien" definido por los extranjeros, al igual que la autoconstrucción de casas, se presenta como un proyecto no acabado que busca realizarse continuamente. Tal aspecto está claramente expresado en las palabras de Robert, un haitiano de alrededor de cuarenta años, quien trabajaba como vendedor en una tienda de Colina. Al preguntarle cuánto tiempo había demorado en construir su vivienda, señaló, de manera apresurada: "No, pero todavía no he terminado" “¿Qué le falta?”, retrucamos. "Falta algo todavía; faltan muchas cositas", dijo de manera un tanto ambigua, como no teniendo certezas sobre lo que construiría en un futuro cercano. Luego, cerró su opinión afirmando:

En cada momento usted coge algo [...] si no es por los materiales [faltantes], se puede hacer entre una semana. Usted puede terminar todo dentro de una semana. Pero puede comprar algo-porque hay cosas que puedes comprar-, pero también hay cosas que usted mismo puede hacer. Todos los meses, cuando usted consigue algo [un material faltante], usted mete algo.

En tanto ejecución en permanente desarrollo, la autoconstrucción modela las prácticas cotidianas del habitar en el campamento y le otorga un carácter procesual a la constitución de los extranjeros como ciudadanos. Este aspecto queda también demostrado en el testimonio de Ana Luz, una mujer dominicana sobre los treinta ańos que se empleaba ocasionalmente en el servicio doméstico. Ante la pregunta por el futuro de la ocupación, ella manifestó que "Bueno, mira, el gusto mío es que me dejaran aqui [en el campamento] porque aqui estoy amplia, tengo patio. En una casa que me den no voy a estar asi [...]; si me dejan aqui, yo misma puedo arreglarla y ponerla bonita también". 
Para Ana Luz, su vivienda contaba con todos los elementos materiales necesarios para habitarla. Sin embargo, ella entendía que aún podía ser mejorada, ampliada y embellecida según sus propios criterios estéticos, posibilidad que parecía mucho más restringida en las soluciones habitacionales que ofrece el Estado. De tal manera, la posibilidad de transformación permanente que brinda la autoconstrucción se vislumbraba como un argumento para reivindicar derechos residenciales sobre el espacio que ocupa. Para quienes colaboraron en la investigación, el campamento materializaba, así, sus aspiraciones no acabadas de integración a la sociedad receptora. Tanto la autoconstrucción de viviendas como su reconocimiento como buenos residentes se entendían como proyectos que, al nunca estar concluidos, debían ser cotidianamente realizados.

Ahora bien, ¿¿de qué manera el proyecto de "vivir bien" verbalizado por nuestros informantes daba cuerpo a sus concepciones de la ciudadanía? La mayoría de ellos sostenía que los chilenos de Nueva Esperanza no destinaban recursos económicos a mantener sus viviendas, porque estaban "acostumbrados a vivir mal". Esa forma de vida, de acuerdo a ellos, era el resultado de ciertas disposiciones éticas de personas a las que "no les gusta trabajar", son "viciosos" (drogadictos) y que, como dio a entender Angélica en una cita anterior, estarían acostumbrados a mendigar. Los extranjeros, al contrario, se describían a sí mismos como sujetos autónomos, responsabilizados y autosuficientes -usando las reflexiones sobre el concepto foucaultiano de gobierno que ofrece Nikolas Rose (1999) y Aihwa Ong (2003)-, capaces de dirigir éticamente su conducta. El acto de autoconstruir permanentemente sus casas con atributos estéticos específicos concretizaría la transformación de los extranjeros que ocupan ilegalmente un terreno en residentes dotados de una capacidad de autogobernarse. La autoconstrucción es, para ellos, una acción que moraliza y dignifica. Las características materiales de las casas y su construcción continua, sin embargo, no solo les ayudan a articular juicios éticos por los que los extranjeros distinguen entre buenos (ellos) y malos (los chilenos) ocupantes. Al mismo tiempo, estas propiedades les permiten formular un tipo "urbano" de ciudadanía en el cual ellos logran reconocerse como ciudadanos constructores de ciudad.

\section{Formando ciudadanos urbanos}

“¿Crees que los extranjeros tienen los mismos derechos que los chilenos?”, era una pregunta que frecuentemente hacíamos a nuestros interlocutores para explorar cómo ellos imaginaban su pertenencia política a la sociedad chilena. La respuesta de Adrián fue, en este contexto, particularmente reveladora por cuanto su autopercepción como sujeto de derecho, aunque tensionada por su condición de inmigrante, estaba íntimamente ligada a su reconocimiento subjetivo como autoconstructor:

Como le digo, para mí [un extranjero] sí tiene derechos, pues es que todos somos personas... [Sin embargo], yo no puedo tener los mismos derechos que usted aqui, porque este es su pais. Pero yo creo que, si yo me porto bien, soy una buena persona, creo que puedo ser un chileno-dominicano, como me ponen en el carnet [...] siempre y cuando usted venga dispuesto a trabajar, no a robar y no a bregar con drogas [...]

Entrevistador: ¿Usted cree que tiene derecho a construir una vivienda aquí en este campamento?

Bueno, yo creo que ahora mismo esto [es] de uno porque uno vive aqui, tenemos una casa ya. Yo digo que yo soy dueño de esto ya. Todos los que vivimos aqui tenemos derechos porque tenemos una casa parada. $Y$ después uno tiene una casa parada en 
un terreno, aunque no sea de uno, ya uno es dueño. Para mí, derecho mio es la casa, porque la tenemos hecha.

Adrián, así como el resto de los extranjeros con quienes trabajamos, articulaban una comprensión paradójica, incluso contradictoria, sobre los derechos y la ciudadanía que se resolvía en la afirmación de la autoconstrucción como una práctica que, en ciertas circunstancias, posibilita la conformación de ciudadanos urbanos moralmente sólidos. Un primer aspecto de este carácter paradójico está en la consideración de los derechos como una prerrogativa universal. Nuestros entrevistados sostenían que todos los individuos, independiente de su origen nacional, tienen derechos en virtud de su condición humana. Desde esta perspectiva universalista de los derechos, el hecho mismo de ser una "persona" concedería derechos a todos los habitantes de la ciudad, incluso no contando con el estatus formal de ciudadano. Sin embargo, esta capacidad para ejercer los derechos era entendida como diferenciada. Los extranjeros, Adrián aseguraba, no pueden tener los mismos derechos que los chilenos porque, para estos últimos, los derechos están constitucionalmente garantizados, no así en el caso de los inmigrantes.

Esta consideración de los derechos como una prerrogativa universal que está desigualmente distribuida dado el distinto estatus legal de las personas parecía, no obstante, subordinarse al carácter moral de sus prácticas cotidianas. En términos simples, esto implicaba que, al momento de pensarse como sujetos de derecho, los extranjeros recurrían principalmente al virtuosismo de sus acciones cotidianas como residentes de un campamento, más que a su condición abstracta de "persona" o "ser humano". Tal perspectiva se revela patentemente cuando nuestros interlocutores interpretan su capacidad para demandar derechos como una práctica sostenida en el acto de autoconstruir la ciudad. Esto explica por qué Adrián indica que los ocupantes ilegales ganan derechos sobre sus espacios residencial una vez que la casa está "parada". En tanto manifestación de lo que James Holston (2008, p. 260) llama contributor rights, los extranjeros legitimaban su capacidad de ejercer derechos a partir de la contribución práctica que, en su vida cotidiana, realizan a la ciudad. Para nuestros informantes, esta contribución presentaba orientaciones éticas específicas que los distinguían de sus pares chilenos: si los extranjeros han adquirido derechos, no es únicamente porque han autoconstruido, sino que también porque -citando a Adrián- "se portan bien" y son "buenas personas". De este modo, asumían que sus acciones cotidianas, en tanto autoconstructores responsables, comprometidos y sacrificados, los dotaban del derecho a tener derechos.

Los extranjeros residentes de Nueva Esperanza, por tanto, configuraban sus concepciones de los derechos sobre la base de una formulación "urbana” de la ciudadanía (Holston, 2008, 2019; Pérez, 2017). En ella, mientras la ciudad era vista como la comunidad política principal, la residencia asumía como criterio central de membresía política. Esta modalidad de imaginarse como legítimos portadores de derechos no se restringía a la afirmación de la condición humana de los migrantes ni al reconocimiento legal como ciudadanos. Más bien, nacía de la constatación de que $s u$ autoconstrucción -en tanto práctica cualitativamente distinta a la que ejecutan los chilenos- era una de las múltiples contribuciones que, como buenos ciudadanos, realizaban los extranjeros. 


\section{Conclusiones}

Las prácticas de construcción de ciudad de los extranjeros que habitan en campamentos han hecho posible la aparición de una ciudadanía urbana en la que ellos evalúan ética y estéticamente sus condiciones de vida como ocupantes ilegales. Si esta formulación de ciudadanía es la forma que encuadra su ejercicio de derechos, el contenido de su demanda por estos deriva del conjunto de prácticas éticas (acciones sobre el yo y sobre los otros) a partir de las cuales estos habitantes urbanos se construyen como sujetos autónomos y responsabilizados. En este proceso, la autoconstrucción permite la transformación de los extranjeros en ciudadanos capaces de ejercer derechos solo cuando los resultados materiales de esa práctica y su continuidad en el tiempo se ajustan a sus ideas de lo que constituye una "buena persona" capaz de "vivir bien". En otras palabras, los extranjeros conciben el proceso de convertirse en miembros de derecho pleno como directamente anclado a su constitución como "buenos ciudadanos", lo que se materializa en la construcción de espacios residenciales con atributos estéticos específicos. Así, los inmigrantes usan $s u$ autoconstrucción para establecer una frontera simbólica entre sus condiciones de vida y las imágenes comúnmente asociadas al habitar informal en el campamento, las que lo representan como un espacio precario, sucio y descuidado.

El caso de análisis discutido en este artículo nos invita a reflexionar sobre los alcances políticos de los campamentos en el Chile contemporáneo, las formas de ciudadanía que allí se gestan y los mecanismos de distribución de derechos que, a través de sus prácticas residenciales, imaginan los migrantes. Los nuevos residentes extranjeros de estas ocupaciones, en contraste con los pobladores chilenos de mediados del siglo XX (Espinoza, 1988; M. Garcés, 2002; Murphy, 2015; Angelcos y Pérez, 2017), no visualizan la autoconstrucción como una acción política subversiva, sino más bien como una expresión tangible de su no reconocimiento como ciudadanos. De tal manera, para facilitar su integración a la sociedad chilena como ciudadanos "honestos" y "sacrificados", los extranjeros se sienten compelidos, en términos simbólicos, a "salir" del campamento a pesar de que, en efecto, vivan dentro de sus límites. De allí que, para afirmar su identidad como sujetos responsabilizados capaces de demandar derechos a través de categorías morales como el esfuerzo y el sacrificio (cf. Pérez, 2018), construyan formas de ciudadanía que distinguen de manera clara a los residentes del campamento, sobre la base de una evaluación ética y estética de sus formas de habitar y autoconstruir el territorio. Del mismo modo que la autoconstrucción, desde su constitución como ciudadanos, presenta una temporalidad específica marcada por la continuidad, persistencia y prolongación en el tiempo. $\mathrm{Al}$ igual que las casas erigidas, el convertirse en ciudadanos se concibe como una operación inacabada e incompleta, que debe ser renovada mediante una redefinición constante de lo que implica "vivir bien". Así, la promesa de alcanzar la propiedad de la vivienda y su reconocimiento como ciudadanos pasa tanto por la práctica cotidiana de la autoconstrucción como por el ejercicio permanente y constante de derechos.

Esta ciudadanía basada en la residencia, en tanto forma localizada y "desnacionalizada" de ciudadanía (Sassen, 2002), revela el carácter estratégico de las ciudades para redefinir los límites y clausuras de la ciudadanía moderna. En efecto, la desnacionalización de los reclamos por derechos expuestos aquí forma parte de un panorama global de mutaciones de la ciudadanía (Ong, 2016). En él, la irrupción de nuevas movilizaciones por derechos -tal como las que reemergieron en octubre de 2019- ocurre en escenarios donde conviven dos racionalidades antagónicas: por un lado, regímenes neoliberales mucho menos proclives a la ampliación de derechos sociales; $y$, por otro, crecientes exigencias por derechos universales, mediante los cuales se busca asegurar condiciones mínimas de existencia para todos los miembros de la 
comunidad política. Ello ha llevado a la conformación global de diversos y contradictorios ensamblajes de ciudadanía. En tal sentido, este artículo exhorta a la investigación de estas formas desnacionalizadas de ciudadanía a trascender del binomio inclusión/exclusión en la sociedad receptora, abriendo la mirada a las (nuevas) formas de imaginación política que se producen a partir de los procesos migratorios y sus anclajes en/sobre ciudades neoliberales. En esa tarea, uno de los aspectos discutidos en este trabajo refiere a la idea de los extranjeros de concebir los derechos menos como prerrogativas universales que como una capacidad "diferenciada" que solo algunos pueden ejercer (cf. Young, 1989; Holston 2011). Ello, ciertamente, cuestiona las premisas básicas en las que se ha apoyado la demanda creciente por derechos sociales en la sociedad chilena, al tiempo que podría profundizar el lenguaje meritocrático que utilizan los sectores conservadores para negar la existencia de derechos universales. No obstante, resulta sugerente observar que, en un segmento de los migrantes residentes en el país, las categorías morales mediante las cuales significan sus vivencias cotidianas les permite concebir una forma de ciudadanía en la que pueden ejercer derechos de ciudadanía más allá de las restricciones de la membresía nacional.

\section{Agradecimientos}

Los autores agradecen el apoyo del Proyecto FONDECYT 1210743, el Proyecto Anillos ANID-PIA SOC180033 y del Centro de Estudios de Conflicto y Cohesión Social ANID/ FONDAP 15130009.

\section{Referencias citadas}

Anderson, B. (1991). Imagined Communities: Reflections on the Origin and Spread of Nationalism. New York, NY: Verso.

Angelcos, N. y Pérez, M. (2017). De la “Desaparición” a la Reemergencia: Continuidades y Rupturas del Movimiento de Pobladores en Chile. Latin American Research Review, 52(1), 94-109.

Atisba (2018). Atisba monitor. El mapa de la inmigración en Santiago. http://www.atisba.cl/wp-content/ uploads/2018/07/Reporte-Atisba-Monitor_Mapa-Inmigraci\%C3\%B3n-en-Santiago.pdf

Bauböck, R. (2003). Reinventing Urban Citizenship. Citizenship Studies, 7(2), 139-160.

Benhabib, S. (2002). The Claims of Culture: Equality and Diversity in the Global Era. Princeton, NJ: Princeton University Press.

Blokland, T., Hentschel, C., Holm, A., Lebuhn, H., y Margalit, T. (2015). Urban Citizenship and Right to the City: The Fragmentation of Claims. International Journal of Urban and Regional Research, 39(4), 655-665.

Brain, I., Prieto, J. y Sabatini, F. (2010). Vivir en Campamentos: ¿Camino hacia la vivienda formal o estrategia de localización para enfrentar la vulnerabilidad? EURE, 36(109), 111-141.

Caldeira, T. (2015). Social Movements, Cultural Production, and Protests: São Paulo’s Shifting Political Landscape. Current Anthropology, 56(S11), S126-S136.

Caldeira, T. (2017). Peripheral urbanization: Autoconstruction, transversal logics, and politics in cities of the global south. Environment and Planning D: Society and Space, 35(1), 3-20. https://doi. org/10.1177/0263775816658479 
Castells, M. (1983). The City and the Grassroots: A Cross-Cultural Theory of Urban Social Movements. Berkeley, CA: University of California Press.

CES-TECHO, C. de E. S. (2021). Catastro Nacional de Campamentos 2020-2021. Santiago de Chile: TE$\mathrm{CHO}$ y Fundación Vivienda.

CIS-TECHO (2015). Datos duros de una realidad más dura: Informe Encuesta Nacional de Campamentos 2015. Santiago de Chile: TECHO-Chile.

Cofré, B. (2011). El Movimiento de Pobladores en el Gran Santiago: Las Tomas de Sitios y Organizaciones en los Campamentos. 1970-1973. Tiempo Histórico, 2, 133-157.

Contreras, Y., Ala-Louko, V. y Labbé, G. (2015). Acceso exclusionario y racista a la vivienda formal e informal en las áreas centrales de Santiago e Iquique. Polis, 14(42), 53-78.

Curahua, D. (2013). Integración social y protección de los derechos humanos de los migrantes. En Migraciones: Conferencia internacional sobre migraciones y Derechos Humanos: Estándares y prácticas (pp. 43-48). Santiago de Chile: Editorial Aún Creemos en los Sueños.

Das, V. (2011). State, citizenship, and the urban poor. Citizenship Studies, 15(3-4), 319-333.

Espinoza, V. (1988). Para una Historia de los Pobres de la Ciudad. Santiago de Chile: Ediciones SUR.

Garcés, A. (2012). Localizaciones para una espacialidad. Territorios de la migración peruana en Santiago de Chile. Chungara. Revista de Antropologia Chilena, 44(1), 163-175.

Garcés, A. y Maureira, M. (2018). De familia a organización étnica: Redes para una espacialidad transfronteriza en la Puna de Atacama. Revista Chilena de Antropología, 37, 230-248.

Garcés, M. (2002). Tomando su sitio: El movimiento de pobladores de Santiago, 1957-1970. Santiago de Chile: LOM Ediciones.

Glaser, B. y Strauss, A. (2017). The Discovery of Grounded Theory: Strategies for Qualitative Research. New York, NY: Routledge.

Guizardi, M. y Garcés, A. (2013). Circuitos migrantes: Itinerarios y formación de redes migratorias entre Perú, Bolivia, Chile y Argentina en el norte grande chileno. Papeles de Población, 19(78), 65-110.

Holston, J. (2008). Insurgent Citizenship: Disjunctions of Democracy and Modernity in Brazil. Princeton, NJ: Princeton University Press.

Holston, J. (2011). Contesting privilege with right: The transformation of differentiated citizenship in Brazil. Citizenship Studies, 15(3-4), 335-352.

Holston, J. (2019). Metropolitan rebellions and the politics of commoning the city. Anthropological Theory, 19(1), 120-142. https://doi.org/10.1177/1463499618812324

Holston, J. y Appadurai, A. (1996). Cities and Citizenship. Public Culture, 8(2), 187-204.

Imilan, W. (2014). Restaurantes peruanos en Santiago de Chile: Construcción de un paisaje de la migración. Revista de Estudios Sociales, 35(48), 15-28.

Imilan, W., Garcés, A.y Margarit, D. (2014). Introducción: Flujos migratorios, redes y etnificaciones urbanas. En Imilan, W., Garcés, A. y Margarit, D. (Eds.). Poblaciones en movimiento. Etnificación de la ciudad, 
redes e integración (pp. 19-38). Santiago de Chile: Ediciones Universidad Alberto Hurtado.

Instituto Nacional de Estadísticas, INE. (2019). Estimación de población extranjera en Chile según pais de nacionalidad. Gobierno de Chile. https:/www.ine.cl/docs/default-source/demografia-y-migracion/ publicaciones-y-anuarios/migraci\%C3\%B3n-internacional/estimaci\%C3\%B3n-poblaci\%C3\%B3n-extranjera-en-chile-2018/ine-dem_estimaci\%C3\%B3n-poblaci\%C3\%B3n-extranjera-en-chile_regiones_ nacionalidad_(2019).pdf?sfvrsn=375441b6_7

Instituto Nacional de Estadísticas, INE (2020). Estimación de personas extranjeras residentes habituales en Chile al 31 de diciembre 2019. Gobierno de Chile. https://www.ine.cl/docs/ default-source/demografia-y-migracion/publicaciones-y-anuarios/migraci\%C3\%B3n-internacional/estimaci\%C3\%B3n-poblaci\%C3\%B3n-extranjera-en-chile-2018/estimaci\%C3\%B3npoblaci\%C3\%B3n-extranjera-en-chile-2019-metodolog\%C3\%ADa.pdf?sfvrsn=5b145256_6

Jirón, P. y Mansilla, P. (2014). Las consecuencias del urbanismo fragmentador en la vida cotidiana de habitantes de la ciudad de Santiago de Chile. EURE, 40(121), 5-28.

Kipnis, A. (2004). Anthropology and the theorisation of citizenship. The Asia Pacific Journal of Anthropo$\log y, 5(3), 257-278$.

Kymlicka, W. (1995). Multicultural Citizenship: A Liberal Theory of Minority Rights. Oxford: Oxford University Press.

Lebuhn, H. (2013). Local border practices and urban citizenship in Europe. City, 17(1), 37-51.

Lefebvre, H. (1996). Writings on Cities. Cambridge, MA: Wiley-Blackwell.

López-Morales, E., Flores, P. y Orozco, H. (2018). Inmigrantes en campamentos en Chile: ¿̨mecanismo de integración o efecto de exclusión? Revista INVI, 33(94), 159-185.

Luque, J. C. (2004). Transnacionalismo y enclave territorial étnico en la configuración de la ciudadanía de los inmigrantes peruanos en Santiago de Chile. Revista Enfoques, 3, 81-102.

Mangin, W. (1967). Latin American squatter settlements: A problem and a solution. Latin American Research Review, 2(3), 65-98.

Margarit, D. y Bijit, K. (2014). Barrios y población inmigrantes: El caso de la comuna de Santiago. Revista INVI, 29(81), 19-77.

Margarit, D. y Galaz, C. (2018). Espacios barriales y convivencia: Reflexiones sobre las concentraciones de población inmigrada y la territorialidad urbana. Revista Rumbos, 17, 23-50.

Márquez, F. (2014). Inmigrantes en territorios de frontera: La ciudad de los otros. Santiago de Chile. EURE, 40(120), 49-72.

Marshall, T. H. (1977). Class, Citizenship, and Social Development. Chicago, IL: University of Chicago Press.

Murphy, E. (2015). For a Proper Home: Housing Rights in the Margins of Urban Chile, 1960-2010. Pittsburgh, PA: University of Pittsburgh Press.

Ong, A. (2003). Buddha Is Hiding: Refugees, Citizenship, the New America. Berkeley, CA: University of California Press. 
Ong, A. (2016). Mutations in Citizenship. Theory, Culture \& Society, 23(2-3), 499-531. https://doi. org/10.1177/0263276406064831

Ortiz, D. (2019, junio 15). Dominicano migrante: "Atravesé un campo minado en la frontera para ingresar a Chile". Interferencia. https://interferencia.cl/articulos/dominicano-migrante-atravese-un-campo-minado-en-la-frontera-para-ingresar-chile

Pavez, J. (2016). Afecciones afrocolombianas: Transnacionalización y racialización del mercado del sexo en las ciudades mineras del norte de Chile. Latin American Research Review, 51(2), 24-45.

Pérez, M. (2017). Reframing housing struggles: Right to the city and urban citizenship in Santiago, Chile. City, 21(5), 530-549. https://doi.org/10.1080/13604813.2017.1374783

Pérez, M. (2018). Toward a life with dignity: Housing struggles and new political horizons in urban Chile. American Ethnologist, 45(4), 508-520. https://doi.org/10.1111/amet.12705

Postero, N. (2007). Now We Are Citizens: Indigenous Politics in Postmulticultural Bolivia. Stanford, CA: Stanford University Press.

Rojas, N. y Silva, C. (2016). La migración en Chile: Breve reporte y caracterización. Madrid: Observatorio Iberoamericano sobre Movilidad Humana, Migraciones y Desarrollo.

Rose, N. (1999). Powers of Freedom: Reframing Political Thought. Cambridge: Cambridge University Press.

Santa María, I. (1973). El desarrollo urbano mediante los “asentamientos espontáneos”: El caso de los “campamentos" chilenos. EURE, 3(7), 103-112.

Sassen, S. (2002). Towards Post-National and Denationalized Citizenship. En Isin, E. F. y Turner, B. S. (Eds.). Handbook of Citizenship Studies (pp. 277-291). Thousand Oaks, CA: SAGE Publications Ltd.

Schiappacasse, P. (2008). Segregación residencial y nichos étnicos de los inmigrantes internacionales en el Área Metropolitana de Santiago. Revista de Geografía Norte Grande, 39, 21-38.

Sheehan, M. (2018). Migrant Residents in Search of Residences: Locating Structural Violence at the Interstices of Bureaucracies. Conflict and Society, 4(1), 151-166. https://doi.org/10.3167/arcs.2018.040112

Spruyt, H. (1994). The Sovereign State and Its Competitors: An Analysis of Systems Change. Princeton, NJ: Princeton University Press.

Stefoni, C. (2013). Comprensión de la discriminación desde la construcción de la diferencia. En Migraciones: Conferencia internacional sobre migraciones y Derechos Humanos: Estándares y prácticas (pp. 55-60). Santiago de Chile: Editorial Aún Creemos en los Sueños.

Stefoni, C. (2016). A la espera de un trabajo. Cuando la precariedad laboral determina las formas de habitar el espacio público. En Canales, A. (Ed.). Debates contemporáneos sobre migración internacional: Una mirada desde América Latina (pp. 211-234). Guadalajara: Universidad de Guadalajara.

Stefoni, C. y Fernández, R. (2011). Mujeres inmigrantes en el trabajo doméstico: Entre el servilismo y los derechos. En Stefoni, C. (Ed.). Mujeres inmigrantes en Chile: ¿Mano de obra o trabajadoras con derechos? (pp. 43-72). Santiago de Chile: Ediciones Universidad Alberto Hurtado.

Stefoni, C. y Stang, F. (2017). La construcción del campo de estudio de las migraciones en Chile: Notas de un ejercicio reflexivo y autocrítico. Íconos. Revista de Ciencias Sociales, 58, 109-129. 
Taylor, C. (1992). Politics of Recognition. En Gutmann, A. (Ed.). Multiculturalism and "The Politics of Recognition" (pp. 25-73). Princeton, NJ: Princeton University Press.

Tijoux, M. E. (2013). Niños(as) marcados por la inmigración peruana: Estigma, sufrimientos, resistencias. Convergencia, 20(61), 83-104.

Tijoux, M. E. y Palominos, S. (2015). Aproximaciones teóricas para el estudio de procesos de racialización y sexualización en los fenómenos migratorios de Chile. Polis, 14(42), 247-275.

Turner, J. C. (1968). Housing Priorities, Settlement Patterns, and Urban Development in Modernizing Countries. Journal of the American Institute of Planners, 34(6), 354-363.

Vaccotti, L. (2017). Migraciones e informalidad urbana. Dinámicas contemporáneas de la exclusión y la inclusión en Buenos Aires. EURE, 43(129), 49-70.

Vicuña, J. T. y Rojas, T. (Eds.). (2015). Migración en Arica y Parinacota: Panoramas y tendencias de una región fronteriza. Santiago de Chile: Servicio Jesuita a Migrantes.

Walzer, M. (1989). Citizenship. En Ball, T., Farr, J. y Hanson, R. L. (Eds.). Political Innovation and Conceptual Change (pp. 211-219). Cambridge: Cambridge University Press.

Young, I. M. (1989). Polity and Group Difference: A Critique of the Ideal of Universal Citizenship. Ethics, 99(2), 250-274. 